\title{
Inclusion of mobile phone numbers into an ongoing population health survey in New South Wales, Australia: design, methods, call outcomes, costs and sample representativeness
}

\author{
Margo L Barr ${ }^{1,2^{*}}$, Jason J van Ritten ${ }^{1}$, David G Steel ${ }^{2}$ and Sarah V Thackway ${ }^{1}$
}

\begin{abstract}
Background: In Australia telephone surveys have been the method of choice for ongoing jurisdictional population health surveys. Although it was estimated in 2011 that nearly 20\% of the Australian population were mobile-only phone users, the inclusion of mobile phone numbers into these existing landline population health surveys has not occurred. This paper describes the methods used for the inclusion of mobile phone numbers into an existing ongoing landline random digit dialling (RDD) health survey in an Australian state, the New South Wales Population Health Survey (NSWPHS). This paper also compares the call outcomes, costs and the representativeness of the resultant sample to that of the previous landline sample.
\end{abstract}

Methods: After examining several mobile phone pilot studies conducted in Australia and possible sample designs (screening dual-frame and overlapping dual-frame), mobile phone numbers were included into the NSWPHS using an overlapping dual-frame design. Data collection was consistent, where possible, with the previous years' landline RDD phone surveys and between frames. Survey operational data for the frames were compared and combined. Demographic information from the interview data for mobile-only phone users, both, and total were compared to the landline frame using $x^{2}$ tests. Demographic information for each frame, landline and the mobile-only (equivalent to a screening dual frame design), and the frames combined (with appropriate overlap adjustment) were compared to the NSW demographic profile from the 2011 census using $x^{2}$ tests.

Results: In the first quarter of 2012, 3395 interviews were completed with 2171 respondents (63.9\%) from the landline frame (17.6\% landline only) and 1224 (36.1\%) from the mobile frame (25.8\% mobile only). Overall combined response, contact and cooperation rates were $33.1 \%, 65.1 \%$ and $72.2 \%$ respectively. As expected from previous research, the demographic profile of the mobile-only phone respondents differed most (more that were young, males, Aboriginal and Torres Strait Islanders, overseas born and single) compared to the landline frame responders. The profile of respondents from the two frames combined, with overlap adjustment, was most similar to the latest New South Wales (NSW) population profile.

Conclusions: The inclusion of the mobile phone numbers, through an overlapping dual-frame design, did not impact negatively on response rates or data collection, and although costing more the design was still cost-effective because of the additional interviews that were conducted with young people, Aboriginal and Torres Strait Islanders and people who were born overseas resulting in a more representative overall sample.

Keywords: Sample survey, Mobile phone, Sampling frame

\footnotetext{
* Correspondence: margo.barr@doh.health.nsw.gov.au

${ }^{1}$ Centre for Epidemiology and Evidence, NSW Ministry of Health, 73 Miller Street, North Sydney, Australia

${ }^{2}$ Centre for Statistical and Survey Methodology, University of Wollongong, Wollongong, Australia
}

\section{Biomed Central}

(c) 2012 Barr et al.; licensee BioMed Central Ltd. This is an Open Access article distributed under the terms of the Creative Commons Attribution License (http://creativecommons.org/licenses/by/2.0), which permits unrestricted use, distribution, and reproduction in any medium, provided the original work is properly cited. 


\section{Background}

Because of increasing numbers of mobile-only phone users worldwide, currently estimated to be $30.2 \%$ in the USA [1], 13\% in Canada [2], 14\% - 19\% across the UK countries [3] and 19\% in Australia [4], it has become increasingly difficult to produce unbiased estimates from random digit dialling (RDD) surveys that only target landline phones [5-7]. Consequently there is now substantial international literature on conducting RDD surveys with mobile phone augmentation [8-12] and the American Association for Public Opinion Researchers (AAPOR) Cell Phone Task Force recommended in their latest report (2010) [12]: "Random digit dialling (RDD) surveys without cell phone augmentation should in their methods report how they have produced unbiased estimates without the cell phone only segment".

In Australia landline telephone surveys have been the method of choice for ongoing population health surveys [13-18]. Although the rate of mobile-only phone users was estimated to be nearly $20 \%$ in 2011 [4] the inclusion of mobile-only phone users into these existing landline population health surveys has not occurred. Studies describing the demographic, socio-economic and health profile of mobile-only phone users have been conducted and have shown that mobile-only phone respondents were different to those who had access to a landline phone using face-to-face survey data $[19,20]$ and internet panel data [21].

Two designs for the inclusion of mobile-only phone users into landline RDD surveys have been discussed in the literature: screening dual-frame design and overlapping dual-frame design [6]. The screening dual-frame design attempts to remove any overlap units usually by screening for telephone ownership prior to conducting the survey and then only interviewing mobile-only phone users from the mobile frame. The overlapping dualframe design accounts for the overlap in the weighting by using an average estimator and a compositing factor. The overlapping dual-frame design, although requiring a more complex weighting strategy, has been growing in favour because it has been shown that persons selected through mobile frames (even if they have both mobile and landline phones) differ to persons selected through landline frames [7].

Two pilots using a dual-frame design had also been conducted in Australia by Pennay in 2010 (700 respondents) and Lui et al. in 2011 (335 females respondents aged 18 to 39 years) [22,23]. Pennay [22] provided particularly useful statistics for planning this study including: the expected numbers of telephone numbers required to get an interview in each of the frames (landline 12 numbers and mobile 25 numbers) and the expected percentage of interviews with persons from landline-only phone households in the landline phone frame (14.5\%), and percentage of interviews with mobile-only phone users from the mobile phone frame (27.6\%).

This paper describes the methods used for the inclusion of mobile phone numbers into the New South Wales Population Health Survey (NSWPHS), an existing ongoing landline RDD health survey in an Australian state [13]. This paper also compares the call outcomes, costs and the representativeness of the resultant sample to that of the previous landline sample.

\section{Methods}

\section{Survey methodology}

Since 2002 the health and wellbeing of the New South Wales (NSW) population (7.3 million) has been monitored using the NSWPHS. A representative sample of approximately 15,000 persons are interviewed each year, with equal numbers from each of the strata (health administrative areas) using landline RDD computer assisted telephone interviewing (CATI). The questionnaire includes questions on: health behaviours, health status, social determinants, demographics and phone ownership. The survey has approval from the NSW Population and Health Services Research Ethics Committee. The questionnaires and the data collection methods are available on the survey website [13].

In order to include mobile only phone users into this existing landline RDD health survey an overlapping dualframe design was chosen. This allowed us to examine the representation of the resultant sample for both an overlapping dual-frame design and, by excluding persons with both mobile phones and landline phones from the mobile frame, a screening dual-frame design.

Details about the procedures for sample generation, sample design, eligibility, sample size, questionnaire, data collection, calling protocol, participant selection and probability of selection weighting for the previous years' landline RDD surveys [24-27] as well as for each of the phone frames are shown in Table 1 . As shown in Table 1 the procedures were, where possible, consistent with the previous years' landline RDD surveys and between frames.

\section{Call outcomes and costing}

Operational data for the survey were downloaded. The data included telephone number, number of attempts, details of each attempt (including duration) and final disposition. Although the final disposition codes used for the survey are site specific they can be easily mapped to the AAPOR definitions [28]. These final dispositions were then entered into the AAPOR outcome rate calculator [29] and all AAPOR levels of response, cooperation, refusal and contact rates were calculated from the groupings of the final dispositions for each frame. Overall rates were then calculated as described in the Non-response in RDD Cell phone 
Table 1 Comparison of survey methods, 2011 NSW Population Health Survey and 2012 NSW Population Health Survey

Procedures and 2011 NSW Population Health Survey

Protocols (Landline phone numbers)

Sample generation

Landline RDD sample frame for each of the administrative strata were generated using "best fit" postcodes for the geography (exchange district and charge zone) associated with the Australian

Communications and Media Authority (ACMA) phone number ranges for NSW [25].

The sample was then randomly ordered within each strata and each number was tested using proprietary software [26] to identify valid and invalid numbers. The resulting valid numbers were used for the study.

\begin{tabular}{|c|c|c|c|}
\hline Sample design & $\begin{array}{l}\text { Stratified two-stage cluster sample design, } \\
\text { with: strata defined by health administration } \\
\text { areas; simple random sampling of clusters } \\
\text { (household telephone numbers) within each } \\
\text { stratum; and simple random sampling of } \\
\text { population elements (household residents) } \\
\text { within each cluster. }\end{array}$ & $\begin{array}{l}\text { Same as for previous landline } \\
\text { survey }\end{array}$ & $\begin{array}{l}\text { Two-stage cluster sample design } \\
\text { with simple random sampling of the } \\
\text { mobile telephone numbers (adult } \\
\text { population element) and simple } \\
\text { random sampling of children in } \\
\text { household (child population } \\
\text { elements). }\end{array}$ \\
\hline Questionnaire & $\begin{array}{l}\text { The questionnaire included questions on: } \\
\text { health behaviours, health status, social } \\
\text { determinants, demographics (including } \\
\text { number of adults and children in the } \\
\text { household) and landline phone ownership } \\
\text { ("How many residential telephone numbers } \\
\text { do you have? Do not include mobile } \\
\text { phone numbers or dedicated FAX numbers } \\
\text { or modems."). The actual questions in the } \\
\text { questionnaire are available on the survey } \\
\text { website. }\end{array}$ & $\begin{array}{l}\text { Same as for previous landline } \\
\text { survey except for the addition of } \\
\text { two questions on mobile phone } \\
\text { ownership ("How many mobile } \\
\text { phone numbers do you personally } \\
\text { have?" and "Is/are your residential } \\
\text { telephone number/s listed in the } \\
\text { White pages?") }\end{array}$ & $\begin{array}{l}\text { Same as for previous landline } \\
\text { survey the addition of two } \\
\text { questions on mobile phone } \\
\text { ownership ("How many mobile } \\
\text { phone numbers do you personally } \\
\text { have?" and "Is/are your residential } \\
\text { telephone number/s listed in the } \\
\text { White pages?") }\end{array}$ \\
\hline Sample & $\begin{array}{l}3000 \text { persons per quarter with equal } \\
\text { numbers in each of the strata }\end{array}$ & 2000 persons per quarter & 1000 persons per quarter \\
\hline Ineligible & $\begin{array}{l}\text { Business landline numbers, non-NSW } \\
\text { residential numbers }\end{array}$ & $\begin{array}{l}\text { Same as for previous landline } \\
\text { survey }\end{array}$ & $\begin{array}{l}\text { Business mobile numbers, non-NSW } \\
\text { residential mobile numbers or } \\
\text { mobile numbers owned by a child } \\
\text { under the age of } 16 \text { years. }\end{array}$ \\
\hline Data collection & $\begin{array}{l}\text { Data collection was undertaken using } \\
\text { SAWTOOTH WinCati version } 4.2 \text { and trained } \\
\text { interviewers from the in-house NSW Ministry } \\
\text { of Health's CATI facility. }\end{array}$ & $\begin{array}{l}\text { Same as for previous landline } \\
\text { survey }\end{array}$ & $\begin{array}{l}\text { Same as for previous landline } \\
\text { survey }\end{array}$ \\
\hline Calling protocol & $\begin{array}{l}\text { The interviewers rang the randomly ordered } \\
\text { landline numbers consecutively to try and } \\
\text { contact households and convince the } \\
\text { household and the respondent to } \\
\text { participate in the survey. Up to } 12 \text { attempts } \\
\text { were made to establish contact and if } \\
\text { possible secure an interview with the } \\
\text { selected respondent within a household. }\end{array}$ & $\begin{array}{l}\text { Same as for previous landline } \\
\text { survey }\end{array}$ & $\begin{array}{l}\text { The interviewers rang the randomly } \\
\text { ordered mobile phone numbers } \\
\text { consecutively to try and contact the } \\
\text { owner of the phone. Because } \\
\text { mobile numbers could be located } \\
\text { anywhere in Australia initial calls } \\
\text { were timed to accommodate } \\
\text { different time zones across Australia. } \\
\text { Up to } 12 \text { attempts were made to } \\
\text { establish contact and if possible } \\
\text { secure an interview with the mobile } \\
\text { phone holder. }\end{array}$ \\
\hline Participant selection & $\begin{array}{l}\text { One person from the household was } \\
\text { randomly selected for inclusion in the } \\
\text { survey. If the selected respondent was a } \\
\text { child under the age of } 16 \text { years, a parent } \\
\text { or carer completed the interview on their } \\
\text { behalf. }\end{array}$ & $\begin{array}{l}\text { Same as for previous landline } \\
\text { survey }\end{array}$ & $\begin{array}{l}\text { The mobile phone holder was } \\
\text { selected. If the owner of the mobile } \\
\text { phone was a parent of a child under } \\
16 \text { years of age they were asked at } \\
\text { the end of the interview if they or } \\
\text { the main carers would agree to } \\
\text { being contacted at a later date to } \\
\text { undertake an interview about one } \\
\text { of their children chosen at random. }\end{array}$ \\
\hline
\end{tabular}

\section{NSW Population Health Survey}

\begin{tabular}{|c|c|}
\hline Landline phone numbers & Mobile phone numbers \\
\hline $\begin{array}{l}\text { Same as for previous landline } \\
\text { survey }\end{array}$ & $\begin{array}{l}\text { The RDD mobile sample frame was } \\
\text { developed using all known } \\
\text { Australian mobile prefixes and then } \\
\text { using proprietary software [27] each } \\
\text { number was tested to identify valid } \\
\text { and invalid numbers. A random } \\
\text { sample of valid mobile numbers was } \\
\text { then provided for the study. }\end{array}$ \\
\hline
\end{tabular}

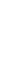

.


Table 1 Comparison of survey methods, 2011 NSW Population Health Survey and 2012 NSW Population Health Survey (Continued)

\begin{tabular}{ll}
\hline Weighting & Adjust for differences in the probabilities of \\
(probability of & selection among subjects (using household \\
selection) & size and number of landline phones in \\
& household).
\end{tabular}

Same as for previous landline

survey except for the inclusion of ratio of landline sample to landline phone populations for each strata.

\author{
Adjust for differences in the \\ probabilities of selection among \\ subjects (using number of mobile \\ phones owned by respondent and \\ ratio of mobile phone sample to \\ mobile phone population and \\ number of children in the \\ household).
}

surveys chapter of the AAPOR Cell Phone Task Force Report [12] using the latest ACMA figures for Australia (5\% landline-only phone users, $19 \%$ mobile-only phone users, and $76 \%$ both mobile phone and landline phone users) [4].

The productivity (phone numbers to get a contact, an eligible contact, and an interview) of the sample for each frame was examined. Call costs (including connection fee, if applicable) and interviewer costs (hourly rate multiplied by the calling time) for each sample frame were also calculated and presented as a cost per completed interview.

\section{Demographic parameter comparisons}

Interview data for the survey were downloaded. The data included a unique identifier, sample frame, strata, and responses to the health behaviours, health status and demographic questions. Demographic information from the mobile frame sample was compared to the landline frame sample using $\chi^{2}$ tests. Demographic information from the mobile frame sample, landline frame sample, combined landline sample with the mobile-only sample (equivalent to a screening dual frame design) and the combined landline sample and mobile sample with appropriate overlap adjustment was compared to the NSW demographic profile from the 2011 census using $X^{2}$ tests.

\section{Results}

In the first quarter of 2012, 3395 interviews were completed with 2171 (63.9\%) being from the landline frame of which 382 (17.6\%) were landlines-only and 1224 (36.1\%) being from the mobile frame of which 316 (25.8\%) were mobile-only.

As shown in Table 2, completed interviews from the mobile frame, compared to the landline frame, were slightly shorter (15.6 minutes v 17.2 minutes), cost 2.3 times more for each completed interview (\$74.42 v $\$ 31.13)$ and required more telephone numbers to obtain a contact ( $2.1 \mathrm{v} 1.9)$, eligible contact $(10.5 \mathrm{v} 7.0)$ and an interview (14.4 v 9.8).

\section{Outcome rates}

Levels of response, contact, cooperation and refusal rates, calculated as per AAPOR definitions, as shown in
Table 2 were similar between frames. Overall combined (with adjustment for the overlap) response, contact, cooperation and refusal rates were $33.1 \%, 65.1 \%, 72.2 \%$ and $17.4 \%$ respectively.

\section{Sample characteristics}

Table 3 shows respondent demographic profiles for the mobile frame (mobile-only, both and total), compared to the landline frame (landline-only, both and total). As shown in Table 3 the demographic profile of the landline frame responders was significantly different to respondents: from the mobile frame who were mobile-only for age group $(\mathrm{p}<0.001)$, sex $(\mathrm{p}=0.049)$, Aboriginality $(\mathrm{p}=0.049)$, country of birth $(\mathrm{p}<0.001)$, and marital status $(\mathrm{p}<0.001)$; from the mobile frame who had both mobile and landline phones for age group $(\mathrm{p}<0.001)$ marital status $(\mathrm{p}=0.003)$ and income $(\mathrm{p}=0.001)$; from the mobile frame for age group $(\mathrm{p}<0.001)$, country of birth $(\mathrm{p}<0.001)$, marital status $(\mathrm{p}<0.001)$ and income $(\mathrm{p}=0.01)$.

Table 4 shows respondent demographic profiles for the landline frame, mobile frame, the landline frame with the mobile-only respondents from the mobile frame, the combined frames (using $\lambda=0.5$ as the compositing factor), and the NSW demographic profile from the 2011 census [30].

As shown in Table 4 the NSW demographic profile was significantly different to respondents: from the landline frame for age group $(\mathrm{p}<0.001)$, sex $(\mathrm{p}=0.037)$, country of birth $(\mathrm{p}=0.02)$, marital status $(\mathrm{p}<0.001)$ and income $(\mathrm{p}=0.015)$; from the mobile frame for age group $(\mathrm{p}=0.03)$ and income $(\mathrm{p}=0.04)$; from the landline frame plus mobile-only phone respondents for age group $(p<0.001)$, marital status $(\mathrm{p}=0.01)$ and income $(\mathrm{p}=0.02)$; and from the combined frame for age group $(p=0.01)$.

\section{Discussion}

When mobile phone numbers were included in the first quarter of 2012 into the NSWPHS using an overlapping dual-frame design, 3395 interviews were completed with just under two thirds from the landline frame and just over one from the mobile frame. Interviews that resulted from the mobile frame, compared to the landline frame, were slightly shorter, cost 2.3 times more for each 
Table 2 Call outcome information and rates for by sample frame and overall (combined)

\begin{tabular}{|c|c|c|c|}
\hline & Landline frame & Mobile frame & Overall \\
\hline $\mathrm{T}=$ Total phone numbers used & 21350 & 17534 & 38884 \\
\hline I=Complete Interviews (1.1) & 2171 & 1224 & 3395 \\
\hline Adults & 1865 & 1085 & 2950 \\
\hline Children & 306 & 139 & 445 \\
\hline $\mathrm{P}=$ Partial & 0 & 0 & 0 \\
\hline R=Refusal and break off (2.1) & 868 & 457 & 1325 \\
\hline NC=Non Contact (2.2) & 660 & 238 & 898 \\
\hline $\mathrm{O}=$ Other $(2.0,2.3)$ & 1163 & 767 & 1930 \\
\hline e: estimated proportion of cases of unknown eligibility that are eligible. & 0.29 & 0.22 & 0.25 \\
\hline UH=Unknown Household (3.1) & 4553 & 5450 & 10003 \\
\hline UO=Unknown other (3.2-3.9) & 0 & 0 & 0 \\
\hline $\mathrm{NE}=$ Not eligible & 11935 & 9462 & 21397 \\
\hline Fax data line $\left(N E_{F}\right)$ & 1352 & 33 & 1385 \\
\hline Non-working number or unusual tone ( $\left.N E_{N W}\right)$ & 2390 & 2637 & 5027 \\
\hline Business, government office, or other organizations $\left(N E_{B}\right)$ & 8100 & 826 & 8926 \\
\hline Not in NSW or mobile owned by child (mobile frame)(NE $)$ & 93 & 5966 & 6059 \\
\hline \multicolumn{4}{|l|}{ Survey length, collection costs and productivity } \\
\hline Average survey length (mins) & 17.2 & 15.6 & \\
\hline Average call costs (per completed interview) & $\$ 7.45$ & $\$ 38.90$ & \\
\hline Average interviewer time costs (per completed interview) & $\$ 23.68$ & $\$ 35.53$ & \\
\hline Total average costs (call costs plus interviewer time costs) & $\$ 31.13$ & $\$ 74.42$ & \\
\hline Telephone numbers used to get a contact: $T /\left(1+R+N E_{1}+N E_{B}\right)$ & 1.9 & 2.1 & \\
\hline Telephone numbers used to get an eligible contact: $T /(I+R)$ & 7.0 & 10.5 & \\
\hline Telephone numbers used to get a completed interview: T/I & 9.8 & 14.4 & \\
\hline \multicolumn{4}{|l|}{ Response Rates } \\
\hline Response Rate 1: $1 /(\mathrm{I}+\mathrm{P})+(\mathrm{R}+\mathrm{NC}+\mathrm{O})+(\mathrm{UH}+\mathrm{UO})$ & $23.1 \%$ & $15.0 \%$ & $18.6 \%$ \\
\hline Response Rate 2: $(\mathrm{I}+\mathrm{P}) /(\mathrm{I}+\mathrm{P})+(\mathrm{R}+\mathrm{NC}+\mathrm{O})+(\mathrm{UH}+\mathrm{UO})$ & $23.1 \%$ & $15.0 \%$ & $18.6 \%$ \\
\hline Response Rate $3: \mathrm{I} /((\mathrm{I}+\mathrm{P})+(\mathrm{R}+\mathrm{NC}+\mathrm{O})+\mathrm{e}(\mathrm{UH}+\mathrm{UO}))$ & $35.1 \%$ & $31.5 \%$ & $33.1 \%$ \\
\hline Response Rate 4: (I+P)/((I+P) + (R+NC+O) +e(UH+UO) ) & $35.1 \%$ & $31.5 \%$ & $33.1 \%$ \\
\hline \multicolumn{4}{|l|}{ Cooperation Rates } \\
\hline Cooperation Rate 1: $1 /(1+P)+R+O)$ & $51.7 \%$ & $50.0 \%$ & $50.7 \%$ \\
\hline Cooperation Rate 2: $(1+P) /((1+P)+R+O))$ & $51.7 \%$ & $50.0 \%$ & $50.7 \%$ \\
\hline Cooperation Rate 3: $1 /((1+P)+R))$ & $71.4 \%$ & $72.8 \%$ & $72.2 \%$ \\
\hline Cooperation Rate 4: $(I+P) /((I+P)+R))$ & $71.4 \%$ & $72.8 \%$ & $72.2 \%$ \\
\hline \multicolumn{4}{|l|}{ Refusal Rates } \\
\hline Refusal Rate 1: R/((I+P)+(R+NC+O) + UH + UO)) & $9.2 \%$ & $5.6 \%$ & $7.2 \%$ \\
\hline Refusal Rate 2: R/((I+P)+(R+NC+O) + e(UH + UO)) & $14.0 \%$ & $11.7 \%$ & $12.8 \%$ \\
\hline Refusal Rate 3: R/((I+P)+(R+NC+O)) & $17.9 \%$ & $17.0 \%$ & $17.4 \%$ \\
\hline
\end{tabular}


Table 2 Call outcome information and rates for by sample frame and overall (combined) (Continued)

\section{Contact Rates}

Contact Rate 1: (I+P)+R+O / (I+P)+R+O+NC+ (UH + UO)

Contact Rate 2: (I+P)+R+O / (I+P)+R+O+NC + e(UH+UO)

$44.6 \%$

$30.1 \%$

$36.5 \%$

Contact Rate 3: (I+P)+R+O / (I+P)+R+O+NC

$68.0 \%$

$62.9 \%$

$65.1 \%$

Notes to Table 2: AAPOR Categories [28] are as follows: Interview $(l)=$ Complete interviews (1.1); Refusal $(R)=$ Respondent refusal (2.112), Household refusal and break off (2.1); Non-contact $(N C)=$ Respondent never available(2.2), away for duration of survey (2.21); Other $(O)=$ Respondent physically or mentally unable to complete interview (2.32), Non-translated language(2.333), Other non-refusal : hang up said nothing/terminated by interviewer/technical problems (2.3):

Unknown Household $(U H)$ = Engaged busy (3.12), No answer (3.13), always answering machine (3.14); Not eligible (NE) = Fax data line (4.2), Non-working number (4.3), unusual tone (4.31), Business, government office, other organizations (4.51), Non-eligible respondent: not in NSW/mobile owned/answered by child (4.7); Calculation of each rate for Overall $=\left(\mathrm{R}_{\mathrm{A}}{ }^{*}\left(\mathrm{~N}_{\mathrm{a}}+\lambda \mathrm{N}_{\mathrm{ab}}^{\mathrm{A}}\right)\right)+\left(\mathrm{R}_{\mathrm{B}}{ }^{*}\left(\mathrm{~N}_{\mathrm{b}}+(1-\lambda) \mathrm{N}_{\mathrm{ab}}^{\mathrm{B}}\right)\right)$ where $\mathrm{R}$ frame rate; $\mathrm{N}$ population proportion; $\lambda=$ overlap adjustment (set 0.5$) ; \mathrm{A}$ landline sample frame; в denotes mobile sample frame; a landline-only phone users;

completed interview and required more telephone numbers to obtain a contact, eligible contact and an interview. Response, contact and co-operation rates were similar between frames. Overall combined response, contact and cooperation rates were $33.1 \%$, $65.1 \%$ and $72.2 \%$ respectively. As expected from previous research [19-23], the demographic profile of the mobile-only phone respondents differed most (more that were young, males, Aboriginal and Torres Strait Islanders, overseas born and single) compared to the landline frame responders. The demographic profile of respondents from the two frames combined, with appropriate overlap adjusted, was most similar to the latest NSW population profile.

Table 3 Comparison of the demographic profile of the mobile frame and the landline frame respondents

\begin{tabular}{|c|c|c|c|c|c|c|c|c|c|c|}
\hline \multicolumn{2}{|c|}{ Demographic group } & \multicolumn{6}{|c|}{ Mobile frame } & \multicolumn{3}{|c|}{ Landline frame } \\
\hline Age groups & $0-15$ & $\begin{array}{c}\begin{array}{c}\text { Mobile } \\
\text { only (\%) }\end{array} \\
8.5\end{array}$ & $\begin{array}{c}\text { p-value } \\
<0.001\end{array}$ & $\begin{array}{l}\begin{array}{c}\text { Both } \\
\text { (\%) }\end{array} \\
12.3 \\
\end{array}$ & $\begin{array}{l}\text { p-value } \\
<0.001\end{array}$ & $\begin{array}{c}\begin{array}{c}\text { Total } \\
\text { (\%) }\end{array} \\
11.4\end{array}$ & $\begin{array}{c}\text { p-value } \\
<0.001\end{array}$ & $\begin{array}{c}\begin{array}{c}\text { Land-line } \\
\text { only (\%) }\end{array} \\
6.0\end{array}$ & $\begin{array}{c}\begin{array}{c}\text { Both } \\
\text { (\%) }\end{array} \\
15.8 \\
\end{array}$ & $\begin{array}{r}\begin{array}{r}\text { Tota } \\
(\%)\end{array} \\
14.1\end{array}$ \\
\hline & $16-24$ & 17.1 & & 10.8 & & 12.4 & & 0.5 & 4.9 & 4.1 \\
\hline & $25-34$ & 41.8 & & 16.6 & & 23.1 & & 1.6 & 6.4 & 5.6 \\
\hline & $35-44$ & 12.3 & & 16.0 & & 15.0 & & 5.2 & 8.0 & 7.6 \\
\hline & $45-54$ & 10.1 & & 19.3 & & 16.9 & & 7.3 & 14.3 & 13.0 \\
\hline & $55-64$ & 7.3 & & 14.9 & & 12.9 & & 16.8 & 22.6 & 21.6 \\
\hline & $65-74$ & 2.5 & & 7.9 & & 6.5 & & 23.3 & 17.3 & 18.4 \\
\hline & 75-high & 0.3 & & 2.2 & & 1.7 & & 39.3 & 10.6 & 15.6 \\
\hline \multirow[t]{2}{*}{ Sex } & Male & 48.4 & 0.049 & 48.3 & 0.052 & 48.4 & 0.052 & 42.9 & 38.0 & 38.9 \\
\hline & Female & 51.6 & & 51.7 & & 51.6 & & 57.1 & 62.0 & 61.1 \\
\hline \multirow[t]{2}{*}{ Aboriginality } & Aboriginal & 5.1 & 0.049 & 1.8 & 0.76 & 2.6 & 0.78 & 2.4 & 2.2 & 2.2 \\
\hline & Non-Aboriginal & 94.9 & & 98.2 & & 97.4 & & 97.6 & 97.8 & 97.8 \\
\hline \multirow[t]{2}{*}{ Country of birth } & Australia & 60.8 & $<0.001$ & 79.4 & 1.00 & 64.9 & $<0.001$ & 76.6 & 80.1 & 79.4 \\
\hline & Overseas & 39.2 & & 20.6 & & 35.1 & & 23.4 & 19.9 & 20.6 \\
\hline \multirow[t]{5}{*}{ Marital status } & Married & 31.3 & $<0.001$ & 61.8 & 0.003 & 54.0 & $<0.001$ & 45.3 & 56.0 & 54.1 \\
\hline & Widowed & 1.9 & & 3.5 & & 3.1 & & 28.7 & 10.5 & 13.7 \\
\hline & Separated & 3.5 & & 3.2 & & 3.3 & & 3.4 & 4.1 & 4.0 \\
\hline & Divorced & 7.4 & & 7.0 & & 7.1 & & 10.8 & 12.6 & 12.3 \\
\hline & Never married & 55.8 & & 24.5 & & 32.5 & & 11.8 & 16.8 & 15.9 \\
\hline \multirow[t]{5}{*}{ Income } & $<\$ 20,000$ & 19.0 & 0.32 & 9.9 & 0.001 & 12.0 & 0.01 & 46.8 & 19.7 & 24.0 \\
\hline & $\$ 20,001-\$ 40,000$ & 14.7 & & 15.7 & & 15.4 & & 24.5 & 18.9 & 19.8 \\
\hline & $\$ 40,001-\$ 60,000$ & 16.8 & & 14.3 & & 14.9 & & 9.3 & 16.2 & 15.1 \\
\hline & $\$ 60,001-\$ 80,000$ & 14.2 & & 13.9 & & 14.0 & & 4.1 & 11.5 & 10.4 \\
\hline & $\$ 80,000$ plus & 35.3 & & 46.3 & & 43.7 & & 15.2 & 33.7 & 30.8 \\
\hline
\end{tabular}

Notes: Chi-squared testing, setting the significance level of $p<0.05$, was used for the comparisons between the mobile phone frame (mobile-only, both and total) sample demographic categories and the total landline frame sample. 
Table 4 Sample comparisons to the latest population profile for NSW

\begin{tabular}{|c|c|c|c|c|c|c|c|c|c|c|}
\hline \multicolumn{2}{|c|}{ Demographic group } & \multicolumn{2}{|c|}{ Landline frame } & \multicolumn{2}{|c|}{ Mobile frame } & \multicolumn{2}{|c|}{ Landline plus mobile only } & \multicolumn{2}{|c|}{ Both frames combined \# } & $\begin{array}{r}2011 \\
\text { Censu }\end{array}$ \\
\hline \multirow{8}{*}{ Age groups } & & & $<0.001$ & & 0.03 & 134 & $<0001$ & 121 & 0.01 & 205 \\
\hline & $16-24$ & 4.1 & & 12.4 & & 5.8 & & 7.3 & & 11.6 \\
\hline & $25-34$ & 5.6 & & 23.1 & & 10.2 & & 13.2 & & 13.6 \\
\hline & $35-44$ & 7.6 & & 15.0 & & 8.2 & & 9.9 & & 14.1 \\
\hline & $45-54$ & 13.0 & & 16.9 & & 12.7 & & 13.4 & & 13.8 \\
\hline & $55-64$ & 21.6 & & 12.9 & & 19.8 & & 17.4 & & 11.7 \\
\hline & $65-74$ & 18.4 & & 6.5 & & 16.4 & & 14.1 & & 7.8 \\
\hline & 75-high & 15.6 & & 1.7 & & 13.5 & & 12.5 & & 6.9 \\
\hline \multirow[t]{2}{*}{ Sex } & Male & 38.9 & 0.04 & 48.4 & 0.85 & 40.1 & 0.07 & 42.8 & 0.20 & 49.3 \\
\hline & Female & 61.1 & & 51.6 & & 59.9 & & 57.2 & & 50.7 \\
\hline \multirow[t]{2}{*}{ Aboriginality } & Aboriginal & 2.2 & 0.86 & 2.6 & 0.94 & 2.6 & 0.96 & 2.6 & 0.96 & 2.5 \\
\hline & Non-Aboriginal & 97.8 & & 97.4 & & 97.4 & & 97.4 & & 97.5 \\
\hline \multirow[t]{2}{*}{ Country of birth } & Australia & 79.4 & 0.02 & 64.9 & 0.42 & 77.1 & 0.07 & 73.4 & 0.30 & 68.6 \\
\hline & Overseas & 20.6 & & 35.1 & & 22.9 & & 26.6 & & 31.4 \\
\hline \multirow[t]{5}{*}{ Marital status } & Married & 54.1 & $<0.001$ & 54.0 & 0.76 & 51.3 & 0.01 & 51.5 & 0.08 & 49.4 \\
\hline & Widowed & 13.7 & & 3.1 & & 12.2 & & 11.1 & & 5.8 \\
\hline & Separated & 4.0 & & 3.3 & & 3.9 & & 3.7 & & 3.1 \\
\hline & Divorced & 12.3 & & 7.1 & & 11.7 & & 10.2 & & 8.3 \\
\hline & Never married & 15.9 & & 32.5 & & 20.9 & & 23.5 & & 33.4 \\
\hline \multirow[t]{5}{*}{ Income* } & $<\$ 20,000$ & 24.0 & 0.02 & 12.0 & 0.04 & 23.4 & 0.02 & 21.9 & 0.05 & 13.7 \\
\hline & $\$ 20,001-\$ 40,000$ & 19.8 & & 15.4 & & 19.2 & & 18.5 & & 19.8 \\
\hline & $\$ 40,001-\$ 60,000$ & 15.1 & & 14.9 & & 15.3 & & 14.7 & & 16.9 \\
\hline & $\$ 60,001-\$ 80,000$ & 10.4 & & 14.0 & & 10.8 & & 11.2 & & 19.8 \\
\hline & $\$ 80,000$ plus & 30.8 & & 43.7 & & 31.3 & & 33.7 & & 29.8 \\
\hline
\end{tabular}

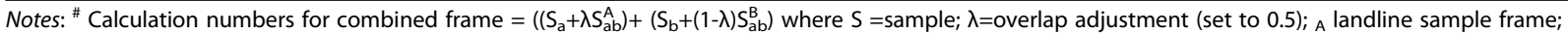
в denotes mobile sample frame; a landline-only phone users; $b$ mobile-only phone users; ab denotes both mobile phone and landline users.

* Census income information was converted from weekly income to annual income for the comparison.

X2 testing, setting the significance level of $\mathrm{p}<0.05$, was used for the comparisons between the sample demographic categories and the population profile (2011 census).

The inclusion of the mobile phone number was logistically very challenging with the biggest challenge being the lack of geography on the mobile frame which resulted in more time and resources being spent on calling ineligible numbers (persons who reside outside NSW). The inclusion of mobile phone numbers in the NSWPHS however is still cost-effective because of the additional interviews that were conducted with young people, Aboriginal and Torres Strait Islanders and people who were born overseas resulting in a more representative sample. This however may not be the case for smaller states where the cost of excluding ineligible (out of state) persons may be prohibitive.

As this study is mainly descriptive there is a need to further examine if using a different overlap adjustment factors would have impacted on the results. Further work also needs to occur with the sample frame provider to minimise the number of invalid and ineligible number (predominantly business numbers) to improve the efficiency of the data collection.

Early results are now becoming available from standalone surveys of the Australian population that are including mobile phone numbers using various designs [31-33] and so we are slowly getting more experience in Australia on conducting RDD surveys with mobile phone augmentation. There is still a need for more detailed methodologies to be provided. So hopefully this study, and the work we are undertaking on weighting strategies for the NSWPHS and an examination of the impact of the design change on the time series, will contribute to a better understanding of how to conduct RDD surveys with mobile phone augmentation in Australia. 


\section{Conclusions}

The inclusion of the mobile phone numbers, through an overlapping dual-frame design, did not impact negatively on response rates or data collection, and although costing more the design was still cost-effective because of the additional interviews that were conducted with young people, Aboriginal and Torres Strait Islanders and people who were born overseas resulting in a more representative overall sample.

\section{Abbreviations}

AAPOR: American Association For Public Opinion Researchers; ACMA: Australian Communications And Media Authority; CATI: Computer assisted telephone interviewing; RDD: Random digit dialling; NSWPHS: New South Wales Population Health Survey.

\section{Competing interests}

The authors declare that they have no competing interests.

\section{Authors' contributions}

MLB developed the overall concepts and planned the study; undertook the analysis and co-wrote the methods and results, wrote the introduction and discussion and finalised the manuscript. JJVR developed and managed the data collection, co-wrote the methods and results, and commented on drafts of the manuscript. DGS provided development and analysis advice and commented on drafts of the manuscript. SVT provided overall support for the study and commented on drafts of the manuscript. All authors read and approved the final manuscript.

\section{Authors' information}

MLB is a PhD student with the Centre for Statistical and Survey Methodology, University of Wollongong, Wollongong, Australia.

\section{Acknowledgments}

We acknowledge the interviewing staff and supervisors at the Centre for Epidemiology and Evidence, NSW Ministry of Health for collecting the data and providing their comments. We also acknowledge the respondents for participating in the survey.

Received: 19 July 2012 Accepted: 22 October 2012

Published: 22 November 2012

\section{References}

1. Blumberg SJ, Luke JV: Wireless substitution: Estimates from the National Health Interview Sunvey. January - June 2011, National Centre for Health Statistics. 2011. http:/www.cdc.gov/nchs/data/nhis/earlyrelease/wireless201112.htm.

2. Statistics Canada, Residential telephone service survey: The Daily, April 5, 2011. http://www.statcan.gc.ca/daily-quotidien/110405/dq110405a-eng.htm.

3. Ofcom research: Ofcom nations and regions tracker, Quarter 1. www.ofcom. org.uk/static/marketdataresearch/statistics/main_set.pdf.

4. Australian Communications and Media Authority (ACMA): Communications report 2010-11 series: Report 2 - Converging communications channels: Preferences and behaviours of Australian communications users. ACMA; 2011.

5. Lee S, Brick JM, Brown ER, Grant D: Growing cell-home population and non-coverage bias in traditional random digit dial telephone health surveys. Health Service Research. 2010, 45(4):1121-1139.

6. Brick JM, Cervantes IF, Lee S, Norman G: Nonsampling errors in dual frame telephone surveys. Survey Methodology 2011, 37(1):1-12.

7. Lynn P, Kaminska O: The impact of mobile phones on survey measurement error, Institute for Social and Economic Research (ISER) Working Paper, Volume 2011-07. Essex: ISER.

8. Kuusela V, Callegaro M, Vehovar V: The influence of mobile telephones on telephone surveys. In Advances in Telephone Survey Methodology. Edited by Lepkowski J, Tucker C, Brick M, De Leeuw E, Japec L, Lavrakas PJ, Link M, Sangste R. Hoboken, NJ: Wiley; 2007:87-112.

9. The American Association for Public Opinion Research (AAPOR): Cell Phone Task Force Report: Guidelines and considerations for survey researchers when planning and conducting RDD and other telephone surveys in the US with respondents reached via cell phone numbers. AAPOR; 2008.
10. Benford R, Tompson T, Fleury C, Feinberg G, Feinberg B, Speulda N, Weber A: Cell phone and landline - considerations for sample design, estimates, weighting, and costs. Hollywood, FL: Paper presented at the 64th annual conference of the American Association for Public Opinion Research; 2009 .

11. Hu SS, Balluz L, Battaglia MP, Frankel MR: Improving public health surveillance using a dual-frame survey of landline and cell phone numbers. Am J Epidemiol 2011, 173:703-711.

12. The American Association for Public Opinion Research (AAPOR): Cell Phone Task Force Report: New considerations for survey researchers when planning and conducting RDD telephone surveys in the US with respondents reached via cell phone numbers. 2010.

13. NSW Population Health Surveys. http://www.health.nsw.gov.au/publichealth/ surveys/index.asp.

14. SA Monitoring and Surveillance System. http://www.health.adelaide.edu.au/ pros/data/samss.

15. Victorian Population Health Survey. http://www.health.vic.gov.au.

16. WA Health and Wellbeing Surveillance System. http://www.health.wa.gov.au.

17. Queensland Health Omnibus Survey. http://www.health.qld.gov.au.

18. ACT General Health Survey. http://www.health.act.gov.au.

19. Grande ED, Taylor AW: Sampling and coverage issues of telephone surveys used for collecting health information in Australia: results from a face-to-face survey from 1999 to 2008. BMC Med Res Methodol 2010, 10:77.

20. Population Health Information Development Unit: Summary report on home phone ownership: Extent and characteristics of the population with no fixed-line phone access. In Audit of Australian Chronic Disease and Associated Risk Factor Data Collections. Edited by Gruszin S, Szuster F. Adelaide: PHIDU; 2010:28-29.

21. Pennay D, Bishop N: Profiling the 'mobile phone only' population: A study of Australians with a mobile phone and no landline telephone, The Social Research Centre Pty Ltd. 2009.

22. Pennay D: Profiling the 'mobile phone only' population: Results from a dualframe telephone survey using a landline and mobile phone sample frame, ASCPRI Social Science Methodology conference proceedings. ASCPRI; 2010

23. Liu B, Brotherton JM, Shellard D, Donovan B, Saville M, Kaldor JM: Mobile phones are a viable option for surveying young Australian women: a comparison of two telephone survey methods. BMC Med Research Methodology 2011, 11:159.

24. Barr M, Baker D, Gorringe M, Fritsche L: NSW Population Health Survey: Description of methods. Sydney: Centre for Epidemiology and Research, NSW Department of Health; 2008. www.health.nsw.gov.au/resources/ publichealth/surveys/health_survey_method.asp.

25. Office of Legislative Drafting and Publishing, Attorney-General's Department: Telecommunications Numbering Plan 1997 including Variation 2007 (No. 5). Canberra: Australian Government; 2008.

26. Sampleworxs Pty Ltd: Household RDD. http://www.sampleworx.com.au/ household_rdd.html.

27. Sampleworxs Pty Ltd: Mobile RDD. http://www.sampleworx.com.au/ mobile_rdd.html.

28. The American Association for Public Opinion Research: Standard definitions: Final dispositions of case codes and outcome rates for surveys. 7th edition. AAPOR; 2011.

29. The American Association for Public Opinion Research: Response rate calculator V3.1 (Excel). http://www.aapor.org/Resources.htm.

30. Australian Bureau of Statistics: Census quickstats. New South Wales: ABS; 2011. http://www.censusdata.abs.gov.au/census_services/getproduct/ census/2011/quickstat/1.

31. Holborn AT, Reavley NJ, Jorm AF: Differences between landline and mobile only respondents in a dual frame mental health literacy survey. ANZJPH 2012, 36:192.

32. Western MC, Pennay D, Haynes M: Results from the first Australian Dual Frame Omnibus Survey. Sydney: Presentation at the Eighth International Conference on Social Science Methodology; 2012.

33. The Social Research Centre: Australian National Preventive Health Agency Research to Inform Key Performance Indicators for the 2011-2015 ANPHA Strategic Plan. Baseline findings; 2011.

doi:10.1186/1471-2288-12-177

Cite this article as: Barr et al:: Inclusion of mobile phone numbers into an ongoing population health survey in New South Wales, Australia: design, methods, call outcomes, costs and sample representativeness. BMC Medical Research Methodology 2012 12:177. 